\title{
Fully Solvable Equilibrium Self-Assembly Process: Fine-Tuning the Clusters Size and the Connectivity in Patchy Particle Systems
}

\author{
Emanuela Bianchi and Piero Tartaglia \\ Dipartimento di Fisica and INFM-CNR-SMC, Università di Roma La Sapienza, Piazzale A. \\ Moro 2, 00185 Roma, Italy \\ Emilia La Nave and Francesco Sciortino* \\ Dipartimento di Fisica and INFM-CNR-SOFT, Università di Roma La Sapienza, Piazzale A. \\ Moro 2, 00185 Roma, Italy
}

Received: June 3, 2007; In Final Form: July 24, 2007

\begin{abstract}
Self-assembly is the mechanism that controls the formation of well-defined structures from disordered preexisting parts. Despite the importance of self-assembly as a manufacturing method and the increasingly large number of experimental realizations of complex self-assembled nano-aggregates, theoretical predictions are lagging behind. Here, we show that for a nontrivial self-assembly phenomenon, originating branched loopless clusters, it is possible to derive a fully predictive parameter-free theory of equilibrium self-assembly by combining the Wertheim theory for associating liquids with the Flory-Stockmayer approach for chemical gelation.
\end{abstract}

\begin{abstract}
Intermolecular self-assembly is the ability of molecules to form supramolecular assemblies ${ }^{1}$ as well as a manufacturing method used to construct aggregate at the nano- or micro-scale, by proper design of the constituent molecules. In the selfassembly bottom-up paradigm, the final (desired) structure is encoded in the shape and properties of the designed building blocks. Realizations of complex self-assembled nano-aggregates ${ }^{2-4}$ have been guided by intuition and sophisticated experimental techniques. A full comprehension of the self-assembly process requires the ability to predict the structures (and their relative abundance) which will be observed in equilibrium as a function of temperature $T$ and density $\rho$, starting from the knowledge of the interparticle interaction potential. Such a request is very much akin to the one that has guided the development of the physics of liquids in the last decades. Differently from the simple liquid case, self-assembly is characterized by a very strong interparticle attraction (significantly larger than the thermal energy $k_{\mathrm{B}} T$ ) and by the fact that the interaction geometry is far from being spherical. The leading "bonding" interaction may indeed be localized in a specific part of the particle surface (patchy interactions ${ }^{5}$ ), it may be active only in the presence of a specific complementary group (lock-and-key interactions, very often encountered in biological self-assembly ${ }^{6-9}$ ), or it may be strongly dependent on the particle orientation. ${ }^{10}$ The presence of strong and patchy interactions poses significant challenges to a parameter-free description of self-assembly. Only equilibrium chain polymerization, the simplest self-assembly process which takes place when bifunctional particles self-assemble into chains of variable length, can be considered to be sufficiently established. ${ }^{11-16}$

In this article, we show that for systems with a small average functionality (but larger than two) it is possible to provide a parameter-free full description of the self-assembly process. We study theoretically and numerically one of the simplest but not
\end{abstract}

\footnotetext{
* Corresponding author.
}

trivial self-assembly processes, namely, a binary mixture of particles with two and three attractive sites. The presence of three (or more)-functional particles, which act as branching points in the self-assembled clusters, introduces two important phenomena which are missing in equilibrium chain polymerization: a percolation transition, where a spanning cluster appears, and a region of thermodynamic instability, the analog of a gas-liquid phase separation. More explicitly, we investigate a binary mixture composed by $N_{2}=5670$ bifunctional particles and $N_{3}=330$ three-functional ones. The resulting average number of sticky spots per particle, that is, the average functionality, is $\langle M\rangle=\left(2 N_{2}+3 N_{3}\right) /\left(N_{2}+N_{3}\right)$. Particles are modeled as hard-spheres of diameter $\sigma$, whose surface is decorated by two (or three) bonding sites at fixed locations. Sites on different particles interact via a square-well potential $V_{\text {SW }}$ of depth $u_{0}$ and attraction range $\delta=0.119 \sigma$. The interaction potential $V(\mathbf{1}, \mathbf{2})$ between particles $\mathbf{1}$ and $\mathbf{2}$ is

$$
V(\mathbf{1}, \mathbf{2})=V_{\mathrm{HS}}\left(\mathbf{r}_{\mathbf{1 2}}\right)+\sum_{i=1, n_{1}} \sum_{j=1, n_{2}} V_{\mathrm{SW}}\left(\mathbf{r}_{\mathbf{1 2}}^{i j}\right)
$$

where $V_{\mathrm{HS}}$ is the hard-sphere potential and $\mathbf{r}_{\mathbf{1 2}}$ and $\mathbf{r}_{\mathbf{1 2}}^{i j}$ are respectively the vectors joining the particle-particle centers and the site-site (on different particles) locations; $n_{i}$ indicates the number of sites of particle $i$. Sites are located on the poles in the case of bifunctional particles and equidistant on the equator in the case of three-functional particles. With this site geometry, the smallest possible bonded ring requires six three-functional particles, while a bonded ring of only bifunctional units requires $\approx 50$ particles. The well width $\delta$ is chosen to ensure that each site, due to steric effect, is engaged at most in one interaction. Distances are measured in units of $\sigma$. Temperature is measured in units of the potential depth (i.e., Boltzmann constant $k_{\mathrm{B}}=$ 1). In the studied model, bonding is properly defined: two particles are bonded when their pair interaction energy is $-u_{0}$. This means that the potential energy of the system is propor-

\subsection{1/jp074281+ CCC: \$37.00 @ 2007 American Chemical Society} Published on Web 09/20/2007 


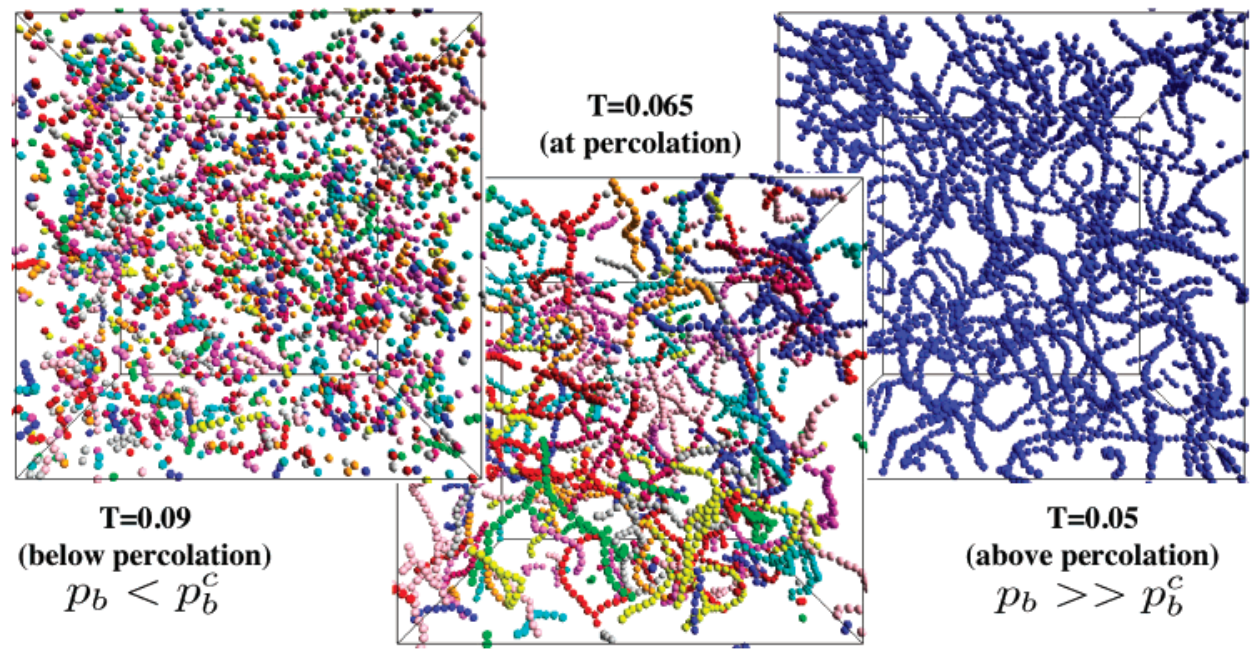

Figure 1. Representation of the studied system for three different temperatures (in units of $u_{0}$ ) at density $\rho \sigma^{3}=0.04$. Equally colored particles belong to the same cluster. The pictures show the structure of the system below percolation (left, $p_{b}<p_{b}^{c}$ ), where particles are aggregated in small finite clusters, at percolation (center, $p_{b} \approx p_{b}^{c}$ ), where a spanning cluster first appears, and well above percolation (right, $p_{b} \gg p_{b}^{c}$ ), where most all of the particles belong to the infinite cluster. The mesh size of the fully connected system, provided by the average length of the chains connecting the three-functional branching points, can be finely controlled by the relative composition of the mixture.

tional to the number of bonds. The lowest energy state of the system (the ground state energy) coincides with configurations in which all bonds are formed, that is, $E_{g s}=-u_{0}\left(2 N_{2}+3 N_{3}\right) /$ 2. As a result, the bond probability, $p_{b}$, can be precisely calculated as the ratio of the potential energy $E$ and $E_{g s}$. Pairs of bonded particles are assumed to belong to the same cluster.

We have performed standard Monte Carlo (MC) Metropolis simulations at several $T$ and $\rho$ (more than 70 different state points). An MC step is defined as an attempted move per particle. A move is defined as a displacement of a randomly selected particle in each direction of a random quantity distributed uniformly between $\pm 0.05 \sigma$ and a rotation around a random axis of a random angle uniformly distributed between \pm 0.1 radiant. Equilibration was monitored via the evolution of the potential energy (a direct measure of the number of bonds in the system). Equilibration at the lowest $T$ required up to $10^{9}$ MC steps (6 months of simulation time). Indeed, at $k_{\mathrm{B}} T / u_{0}=$ 0.05 , a bond breaks in average every $5 \times 10^{8}$ attempts.

The extremely long Monte Carlo simulations provide a numerically "exact" description of the equilibrium self-assembly process in this model. On cooling, particles aggregate in open larger and larger clusters which then coalesce into a spanning structure, eventually incorporating all particles. A pictorial representation of the structure of the system on varying $T$ (below, at, and above percolation) is shown in Figure 1. Clusters are composed by rather stiff chains of bifunctional particles crosslinked by the three-functional ones.

To develop a parameter-free theoretical description of the cluster formation, we combine Wertheim (W) theory ${ }^{17-19}$ for estimating the $T$ and $\rho$ dependence of $p_{b}$ with the FloryStockmayer (FS) predictions for the cluster size distribution in chemical gelation, providing a detailed and predictive theory of reversible self-assembly.

The Wertheim theory, developed back in 1980 for describing the free energy of molecules with fixed valence (associating liquids), can be transferred to particles with a small number of patchy interacting sites. The main assumption in the theory is that molecules (or particles) cluster in open structures without closed bond loops. Such a condition, as we will show in the following, can be realized with patchy particles when the average functionality is small and the chains of bifunctional particles are not significantly flexible. The Wertheim theory predicts that $p_{b}$ can be calculated from the chemical equilibrium between two nonbonded sites forming a bonded pair. For the present model, such relation reads:

$$
\frac{p_{b}}{\left(1-p_{b}\right)^{2}}=\langle M\rangle_{\rho} \Delta
$$

where $\Delta=4 \pi \int g_{\mathrm{HS}}\left(r_{12}\right)\langle f(12)\rangle_{\omega_{1}, \omega_{2}} r_{12}^{2} \mathrm{~d} r_{12}$. Here, $g_{\mathrm{HS}}(12)$ is the reference hard-sphere radial distribution function, $f(12) \equiv$ $\mathrm{e}^{-V_{S W}\left(\mathbf{r}_{12}{ }^{i j) / k_{\mathrm{B}} T}-1\right.}$ is the Mayer $f$ function between two arbitrary sites $i$ and $j$, and $\langle f(12)\rangle_{\omega_{1} \omega_{2}}$ represents an angle average over all orientations of the two particles at fixed relative distance $r_{12}{ }^{20}$ The comparison between the theoretical predictions and the "exact" numerical data for the $T$ and $\rho$ dependence of $p_{b}$ are shown in Figure 2. Data show clearly that the theory is able to predict precisely $p_{b}$ (or equivalently the system potential energy) in a wide $T$ and $\rho$ range. At low $T, p_{b} \rightarrow 1$, and the system approaches a fully bonded disordered (ground state) configuration.

To derive information on the structure of the system and the connectivity of the aggregates, we connect the W and the FS theories. Indeed, the hypothesis of absence of closed bonding loops is at the basis of both theoretical approaches. The $\mathrm{W}$ prediction for $p_{b}$ can thus be consistently used in connection with the FS approach ${ }^{21}$ to predict the $T$ and $\rho$ dependence of the cluster size distributions. In the present case, the number of clusters (per unit volume) containing $l$ bifunctional particles and $n$ three-funtional ones can be written ${ }^{21}$ as

$$
\begin{gathered}
\rho_{n l}=\rho_{3} \frac{\left(1-p_{b}\right)^{2}}{p_{3} p_{b}}\left[p_{3} p_{b}\left(1-p_{b}\right)\right]^{n}\left[p_{2} p_{b}\right]^{l} w_{n l} \\
w_{n l}=3 \frac{(l+3 n-n) !}{l ! n !(n+2) !}
\end{gathered}
$$

where $p_{3} \equiv 3 N_{3} /\left(2 N_{2}+3 N_{3}\right)$ and $p_{2}=1-p_{3}$ are the probabilities that a randomly chosen site belongs to a threefunctional or to a bifunctional particle, $p_{b}$ is given by eq 2 , and $w_{n l}$ is a combinatorial contribution. ${ }^{21}$ Distributions are normalized in such a way that $\sum_{\ln , l+n>0}(l+n) \rho_{n l}=\rho_{2}+\rho_{3}$. As shown in Figure 3 (top) on decreasing $T$, the $\rho_{n l}$ distribution becomes 


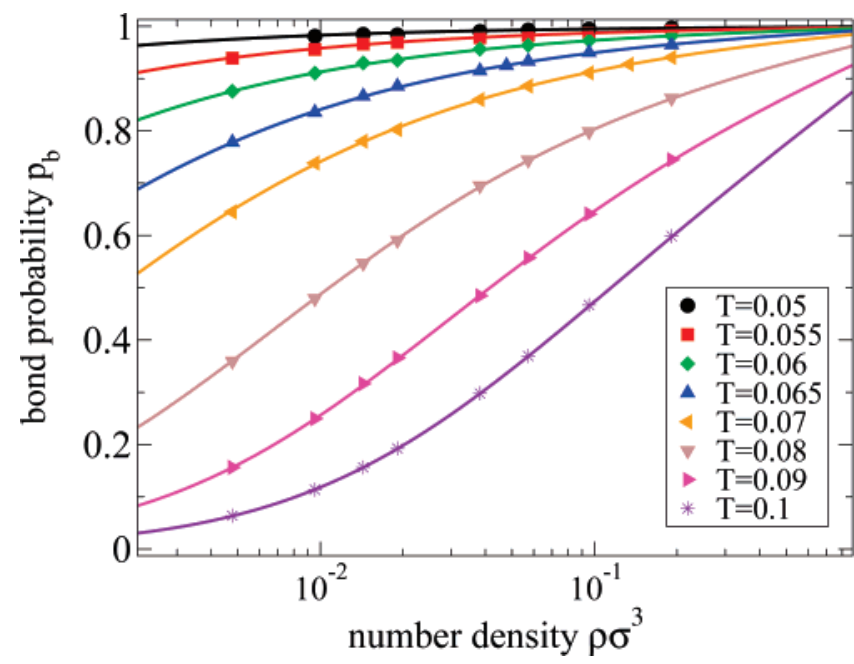

Figure 2. Temperature (in units of $u_{0}$ ) and density dependence of the bond probability $p_{b}$. Points are simulation results based on Monte Carlo simulations for nine different densities and eight different temperatures. Lines are parameter-free predictions based on the Wertheim theory. Note that at low $T$ the system reaches a fully bonded configuration.
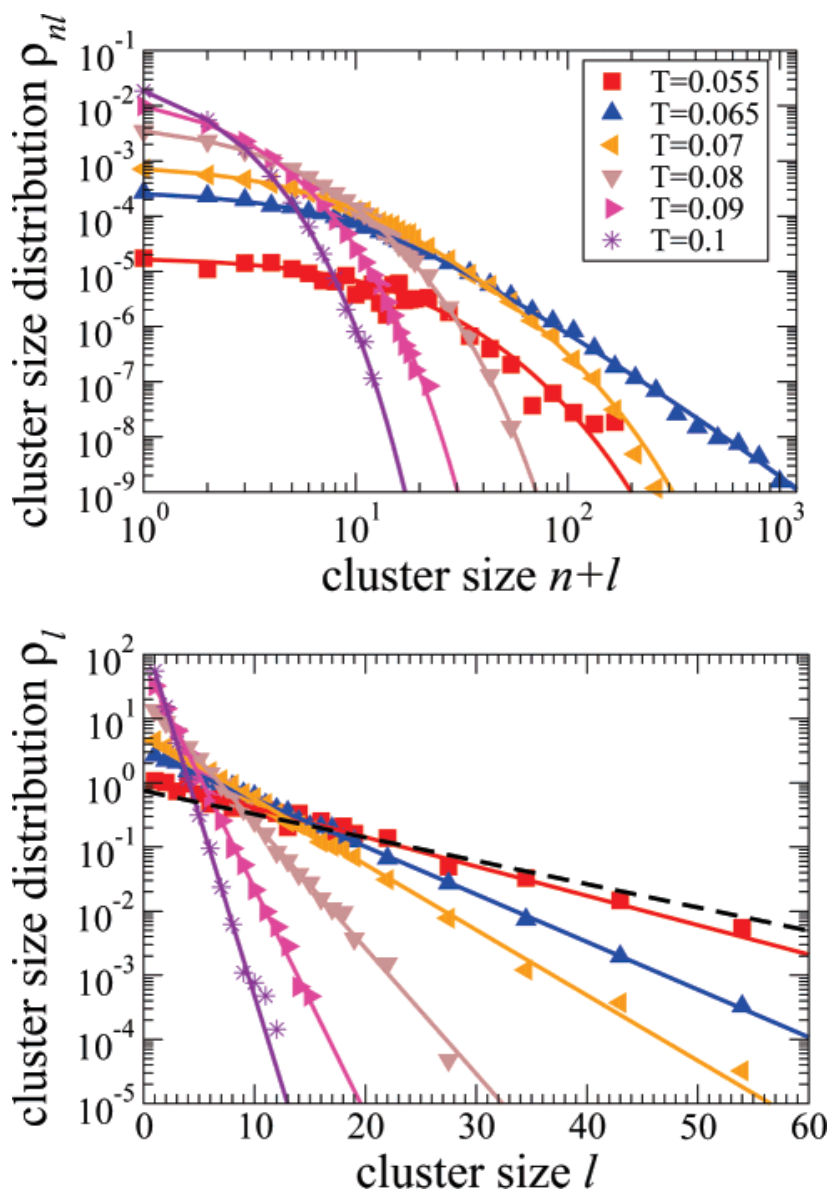

Figure 3. Cluster size distribution for $\rho \sigma^{3}=0.04$ for some of the investigated $T$. Points are simulation data and lines are the corresponding theoretical curves. (top) The number of finite clusters (per unit volume) containing $l$ bifunctional units plus $n$ three-functional ones. (bottom) The number of chains of length $l$ (per unit volume) composed of bifunctional particles. At low $T$, the distribution approaches its limiting (concentration controlled) value $\rho_{l}=\rho_{2} p_{3}^{2} p_{2}^{l-1}$, shown by the dashed line.

wider and wider and develops a power-law tail with exponent -2.5 , characteristic of loopless percolation. ${ }^{22}$ On further decreasing $T$, the distribution of finite size clusters progressively shrinks, since most of the particles attach themselves to the infinite cluster. Data show that eq 3 , with no fitting parameters, predicts extremely well the numerical distributions at all state points, both above and below percolation.

The three-functional particles act as branching points of the network formed by long chains of two-functional particles. Visualizing the structure of the system in term of chains of twocoordinated particles providing a link between the threecoordinates ones, it is possible to predict the number of finite size clusters composed of $n$ three-functional units, irrespective of the number of bifunctional units. The system can thus be considered as a one-component fluid of three-functional particles forming clusters, in which the bonding distance between the three-functional particles is given by the length of the chains formed by the bifunctional units. Following again $\mathrm{FS}$, it is possible to predict the $p_{b}$ value at which the systems develop a percolating structure: when $p_{b} \geq p_{b}^{c} \equiv 1 /\left(1+p_{3}\right)=$ 0.9256 , an infinite cluster is present in the system. The percolation line is thus the locus of points in the phase diagram such that $p_{b}(T, \rho)=p_{b}^{c}$, with $p_{b}(T, \rho)$ given in eq 2 . Along the percolation locus, the product $\rho \Delta\left(\rho \mathrm{e}^{1 / T}\right.$ at low $\left.T\right)$ is constant (from eq 2).

It is also possible to predict the length distribution of chains of bifunctional particles. The number of chains (per unit volume) of length $l$, normalized in such a way that $\sum_{l=1}^{\infty} l \rho_{l}=\rho_{2}$, is

$$
\rho_{l}=\rho_{2}\left(1-p_{2} p_{b}\right)^{2}\left(p_{2} p_{b}\right)^{l-1}
$$

The $\rho_{l}$ distribution is thus always exponential. At low $T$, when $p_{b} \rightarrow 1$, the distribution becomes controlled only by the relative fraction of two-functional particles, providing a method for tuning the porosity of the fully connected percolating structure via the relative composition of the binary mixture. Indeed, when $p_{b} \rightarrow 1$, the average distance $\bar{l}$ between branching points in the network becomes only a function of $p_{2}$; that is, $\bar{l}=1 /\left(1-p_{2}\right)$. Figure 3 (bottom) shows that also the $\rho_{l}$ distribution is perfectly described by the combined W-FS theory.

In the framework of the FS approach, it is also possible to evaluate the number density of clusters $\rho_{c} \equiv \sum_{n l} \rho_{n l}$ as a function of $p_{b}$, irrespective of the cluster size. Below percolation, in the absence of bonding loops, the relation between $\rho_{c}$ and $p_{b}$ is linear, since each added bond decreases the number of clusters by one. Above percolation, the relation crosses to a nonlinear behavior, so that the number of clusters becomes 1 when $p_{b}=$ 1 . Within the FS theory, $\rho_{c}$ can be calculate for all $p_{b}$ values, assuming that finite clusters do not contain closed loops. As shown in Figure 4, the simulation data conform perfectly to the theoretical expectation both below and above percolation. This suggests that, when the average functionality is small and the chains of bifunctional particles have a large persistence length, bonding loops in finite size clusters can be neglected. This agreement, which covers the entire range of $p_{b}$ values, implies that closed loops of bonds are statistically less favored than the corresponding open structure. The relative statistical weight results from the competition between the energy of forming the extra additional bond and the reduction of entropy associated with the closure of the loop. Hence, we interpret the absence of closed loops as resulting from the large configurational entropy of the long bifunctional chains.

To further check the quality of the theory, we numerically evaluate the connectivity properties of each studied state point, searching for the presence of clusters which are infinite under periodic boundary conditions. A state point is considered percolating when, accounting for periodic boundary conditions, 


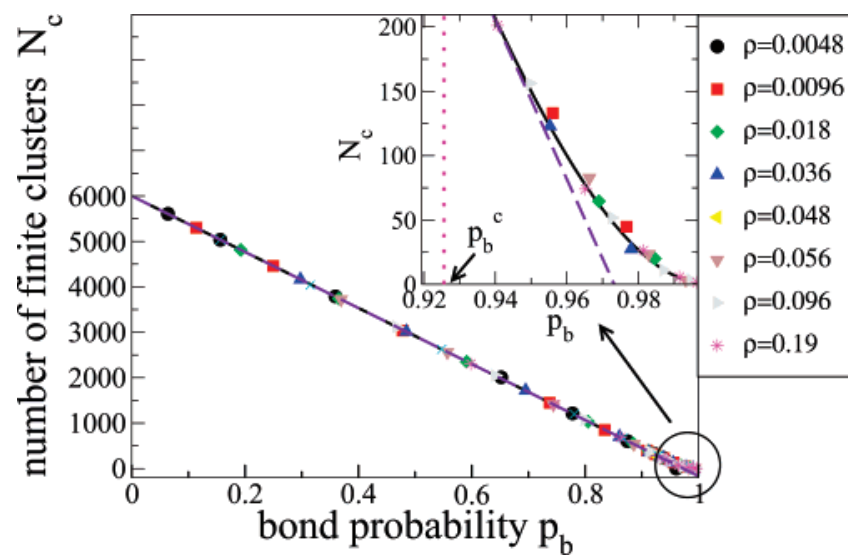

Figure 4. Relation between the number of finite-size clusters $N_{c}$ (irrespective of their size) and the bond probability. Symbols: simulation results for all studied densities. Solid line: Flory-Stockmayer predictions (both below and above percolation). Below percolation, in the absence of bonding loops, $N_{c}$ is given by the difference between the total number of simulated particles $N=6000$ and the number of bonds $N_{b}$, since each added bond decreases $N_{c}$ by one; i.e., $N_{c}=N-$ $N_{b}=N-p_{b}\left(2 N_{2}+3 N_{3}\right) / 2$. This linear relation (dashed line) can be extended above percolation (dotted line) but never beyond the point where $N_{c}<1$. The inset enlarges the region of large $p_{b}$ values, to provide evidence that the FS approach is valid over the entire $p_{b}$ range.

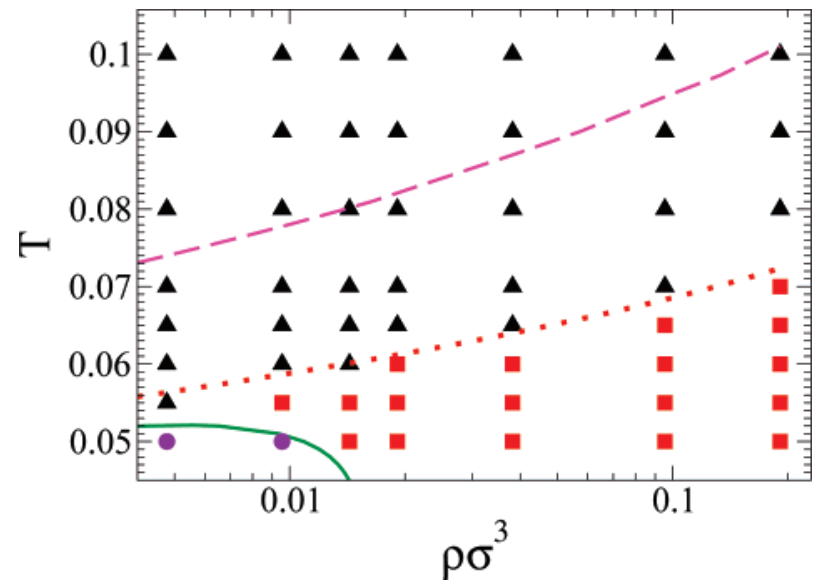

Figure 5. Phase-diagram of the studied model. Lines are theoretical predictions: the solid (green) line is the spinodal curve obtained from the Wertheim equation of state; i.e., $(\partial P / \partial V)_{T}=0$; the dashed (magenta) line is the locus of points such that the specific heat is maximum; i.e., $\left(\partial C_{V} / \partial T\right)_{V}=0$; and the dotted (red) line is the percolation locus $p_{b}(\rho$, $T)=p_{b}^{c}$. Points are simulation results: the (black) triangles are the nonpercolating equilibrium state points, and the (red) squares are the equilibrium percolating state points. Circles are (nonequilibrium) state points characterized by a bimodal distribution of the density fluctuations, indicating a phase-separated structure.

an infinite cluster is present in more than $50 \%$ of the configurations. The resulting partitioning of the state points into percolating and nonpercolating ones (see Figure 5) is fully consistent with the theoretical prediction of the percolation line $p_{b}(T, \rho)$ $=p_{b}^{c}$. The theory also predicts a line of constant volume specific heat $C_{V}$ maxima (also observed in chain polymerization $^{11,23}$ ), provided by the inflection point in the $p_{b}$ versus $T$ curves (Figure 2), which also agrees very well with the simulation results. The line of $C_{V}$ extrema is also shown in Figure 5. The presence of a maximum in $C_{V}$ is a characteristic of bond-driven assembly, and the locus of maxima in the $T-\rho$ plane is one of the precursors of the self-assembly process for low functionality particles.
The $\mathrm{W}$ theory predicts a liquid-gas phase separation at small $\rho$ for any nonvanishing amount of branching points. ${ }^{24,25}$ According to the theory, at low $T$ and sufficiently small $\rho$, the system phase separates into two phases of different density and connectivity. The theoretical spinodal curve, the line separating the stable (or metastable) state points from the unstable ones, is defined as the locus of points such that the volume $V$ derivative of the pressure $P$ vanishes; that is, $(\partial P / \partial V)_{T}=0$. For the present model, it is located below the percolation line, and the two lines merge asymptotically for $T \rightarrow 0$ and $\rho \rightarrow 0$ as shown in Figure 5. The analysis of the numerical configurations for the two investigated state points which happened to be located inside the spinodal are indeed characterized by a bimodal distribution of the density fluctuations and by a very large value of the small-angle structure factor, indicating a phase-separated structure. This confirms that, in the absence of bonded rings, the region of liquid-gas instability can also be predicted using the $\mathrm{W}$ theory. The role of bond rings on the thermodynamic of thermoreversible gels has recently been discussed in ref 15 .

To summarize, this work provides the first fully solvable example of an equilibrium self-assembly process which goes beyond the equilibrium chain polymerization, complementing recent numerical studies and attempts at a direct comparison between theory and simulation in related systems. ${ }^{15,26,27}$ The presence of a non-negligible number of three-valent particles brings in a percolation phenomenon and a gas-liquid instability. These two loci are located in the region of $T$ below the $C_{V}$ maximum, suggesting a cascade of phenomena characterizing the self-assembly process in systems with low $\langle M\rangle$ : onset of bonding $\left(C_{V} \max \right)$, percolation, and eventually at low $\rho$, phase separation. The resulting phase diagram bears strong similarities with the ones discussed in previous mean field ${ }^{28,29}$ and numerical $^{15,26,30}$ studies of thermoreversible gelation.

The possibility of theoretically describing the self-assembly process in the present model, designed to minimize the closedbond loops effects, provides a benchmark for testing novel approaches and approximations accounting for more general cases. The quality of the predictions makes it possible to exploit a fine-tuning of the percolation properties. Using the recently synthesized colloidal particles ${ }^{31,32}$ or functionalizing colloids, ${ }^{33-35}$ we believe it will be possible, by choosing the appropriate $\rho$ and $T$, to generate materials with specific connectivity lengths, to generate gels with desired porosity, or to build on the fractal nature of the self-assembled clusters. The material structure can be made permanent, if needed, by freezing the bonding pattern with a fast cooling process.

Finally, $p_{b}$ appears to be the effective parameter controlling the structure of the system, since configurations at different $\rho$ and $T$ but equal $p_{b}$ are characterized by the same distribution of cluster sizes. Configurations only differ in the relative distance between these clusters and, more interestingly, on the lifetime of the resulting bonds, $\tau \sim \mathrm{e}^{u_{0} / k_{\mathrm{B}} T}$. It is thus in principle possible to decouple the effects related to the persistence of the clusters as units and the dynamics of clusters. The possibility of interpolating in a continuous and structural preserving way from chemical to physical gels may offer a unifying picture of these two apparently different arrest processes.

Acknowledgment. We acknowledge support from MIURPrin and MCRTN-CT-2003-504712.

\section{References and Notes}

(1) Whitesides, G. M.; Boncheva, M. Beyond molecules: Self-assembly of mesoscopic and macroscopic components. Proc. Natl. Acad. Sci. U.S.A. 2002, 99, 4769-4774. 
(2) Tang, Z.; Zhang, Z.; Wang, Y.; Glotzer, S. C.; Kotov, N. A. Selfassembly of CdTe nanocrystals into free-floating sheets. Science 2006, 314, 274-278.

(3) van Blaaderen, A. Colloids get complex. News Views, Nature 2006, 439, 545-546.

(4) Yin, Y.; Xia, Y. Self-assembly of spherical colloids into helical chains with well-controlled handedness. J. Am. Chem. Soc. 2003, 125, 2048-2050

(5) Zhang, Z.; Horsch, M. A.; Lamm, M. H.; Glotzer, S. C. Tethered nano building blocks: toward a conceptual framework for nanoparticles self-assembly. Nano Lett. 2003, 3 (10), 1341-1346.

(6) Fraenkel-Conrat, H.; Williams, R. C. Reconstitution of active tobacco mosaic virus from its inactive protein and nucleic acid components. Proc. Natl. Acad. Sci. U.S.A 1955, 41, 690-698.

(7) Nguyen, H. D.; Reddy, V. S.; Brooks, C. L., III. Deciphering the kinetic mechanism of spontaneous self-assembly of icosahedral capsids. Nano Lett. 2007, 7, 338-344.

(8) Zhang, Z.; Glotzer, S. C. Self-assembly patchy particles. Nano Lett. 2004, 4 (8), 1407-1413.

(9) Workum, V.; Douglas, J. F. Symmetry, equivalence, and molecular self-assembly. Phys. Rev. E 2006, 73, 031502.

(10) Glotzer, S. C.; Solomon, M. J. Anisotropy of buliding blocks and their assembly into complex structures. Nat. Mater. 2007, 6, 557-562.

(11) Greer, S. C. Reversible polymerizations and aggregations. Ann. Rev. Phys. Chem. 2002, 53, 173-200.

(12) Rouault, Y.; Milchev, A. Monte Carlo study of living polymers with the bond-fluctuation method. Phys. Rev. E 1995, 51, 5905.

(13) Wittmer, J. P.; Milchev, A.; Cates, M. E. Dynamical Monte Carlo study of equilibrium polymers: Static properties. J. Chem. Phys. 1998, 109, 834-845.

(14) Dudowicz, J.; Freed, K. F.; Douglas, J. F. Flory-Huggins model of equilibrium polymerization and phase separation in the Stockmayer fluid. Phys. Rev. Lett. 2004, 92, 045502.

(15) Kindt, J. T. Simulation and theory of self-assembled networks: ends, junctions, and loops. J. Phys. Chem. B 2002, 106, 8223-8232.

(16) Sciortino, F.; Bianchi, E.; Douglas, J. F.; Tartaglia, P. Self-assembly of patchy particles into polymer chains: A parameter-free comparison between Wertheim theory and Monte Carlo simulation. J. Chem. Phys. 2007, $126,194903$.

(17) Wertheim, M. S. Fluids with highly directional attractive forces. I. Statistical thermodynamics. J. Stat. Phys. 1984, 35, 19-34.

(18) Hansen, J. P.; McDonald, I. R. Theory of Simple Liquids, 3rd ed.; Academic Press: New York, 2006.
(19) Jackson, G.; Chapman, W. G.; Gubbins, K. E. Phase equilibra of associating fluids: spherical molecule with multiple bonding. Mol. Phys. 1988, 65, 1-31.

(20) Wertheim, M. S. Fluids of dimerizing hard spheres, and fluid mixtures of hard spheres and dispheres. J. Chem. Phys. 1986, 85, 29292936.

(21) Flory, P. J. Principles of Polymer Chemistry; Cornell University Press: London, 1953.

(22) Rubinstein, M.; Colby, R. H. Polymer Physics; Oxford University Press Inc.: New York, 2003.

(23) Dudowicz, J.; Freed, K. F.; Douglas, J. F. Lattice model of living polymerization. I. Basic thermodynamic properties. J. Chem. Phys. 1999, $111,7116-7130$.

(24) Sear, R. P. Phase behavior of a simple model of globular proteins. J. Chem. Phys. 1999, 111, 4800-4806.

(25) Bianchi, E.; Largo, J.; Tartaglia, P.; Zaccarelli, E.; Sciortino, F. Phase diagram of patchy colloids: towards empty liquids. Phys. Rev. Lett. 2006, 97, 168301

(26) Kumar, S.; Douglas, J. F. Gelation in physically associating polymer solution. Phys. Rev. Lett. 2001, 87, 188301.

(27) Kindt, J. T. Simulation and theory of self-assembly and network formation in reversibly cross-linked equilibrium polymers. J. Chem. Phys. 2005, 123, 144901-144911.

(28) Coniglio, A.; Stanley, H. E.; Klein, W. Site-bond correlatedpercolation problem: A statistical mechanical model of polymer gelation. Phys. Rev. Lett. 1979, 87, 518.

(29) Zilman, A. G.; Safran, S. A. Thermodynamics and structure of selfassembled networks. Phys. Rev. E 2002, 66, 051107.

(30) Zaccarelli, E.; Buldyrev, S. V.; La Nave, E.; Moreno, A. J.; SaikaVoivod, I.; Sciortino, F.; Tartaglia, P. Model for reversible colloidal gelation. Phys. Rev. Lett. 2005, 94, 218301.

(31) Manoharan, V. N.; Elsesser, M. T.; Pine, D. J. Dense packing and symmetry in small clusters of microspheres. Science 2003, 301, 483-487.

(32) Cho, Y.-S.; Yi, G.-R.; Lim, J.-M.; Kim, S.-H.; Manoharan, V. N.; Pine, D. J.; Yang, S.-M. Self-organization of bidisperse colloids in water droplets. J. Am. Chem. Soc. 2005, 127, 15968-15975.

(33) Mirkin, C. A.; Letsinger, R. L.; Mucic, R. C.; Storhoff, J. J. A DNA-based method for rationally assembling nanoparticles into macroscopic materials. Nature 1996, 382, 607-609

(34) Harris, N. C.; Kiang, C.-H. Disorder in DNA-linked gold nanoparticle assemblies. Phys. Rev. Lett. 2005, 95, 046101.

(35) Jackson, A. M.; Myerson, J. W.; Stellacci, F. Spontaneous assembly of subnanometre-ordered domains in the ligand shell of monolayer-protected nanoparticles. Nat. Mater. 2004, 3, 330-336. 